\title{
Improved Anti-Triple Negative Breast Cancer Effects of Docetaxel by RGD-Modified Lipid-Core Micelles
}

\author{
Rujing Chen ${ }^{1,2, *}$ \\ Shuting $\mathrm{Ni}^{1}{ }^{1} *$ \\ Wangyan Chen' \\ Mei $\mathrm{Liu}^{3}$ \\ Jianfang Feng ${ }^{4}$ \\ Kaili Hu'
}

'Murad Research Center for Modernized

Chinese Medicine, Institute of

Interdisciplinary Integrative Medicine

Research, Shanghai University of

Traditional Chinese Medicine, Shanghai,

201203, People's Republic of China;

${ }^{2}$ Department of Pharmacy, Ruijin Hospital, Shanghai Jiaotong University

School of Medicine, Shanghai, 200025,

People's Republic of China; ${ }^{3}$ Shanghai

Innovation Center of TCM Health

Service, Shanghai University of Traditional

Chinese Medicine, Shanghai, 20I203,

People's Republic of China; ${ }^{4}$ School of

Pharmacy, Guangxi University of Chinese

Medicine, Nanning, 53000I, People's

Republic of China

*These authors contributed equally to this work

Correspondence: Kaili Hu

Murad Research Center for Modernized

Chinese Medicine, Institute of

Interdisciplinary Integrative Medicine

Research, Shanghai University of

Traditional Chinese Medicine, Shanghai,

20I203, People's Republic of China

Tel +86-2I-5I32-2534

Fax +86-2|-5|32-253|

Email kaili-hu@।63.com

Jianfang Feng

School of Pharmacy, Guangxi University

of Chinese Medicine, Nanning, 53000I,

People's Republic of China

Email fengjianfang@vip.163.com
Purpose: A novel RGD-modified PEGylated lipid-core micelle delivery system was designed to improve the anti-cancer effect of docetaxel on triple negative breast cancer (TNBC).

Methods: The tumor-targeted lipid-core micelles loaded with docetaxel were prepared and characterized. Their morphology, particle size, zeta potential, entrapment efficiency, release profiles, and targeting effects were studied. The antitumor effects of the docetaxel-loaded nano-micelles were investigated in a MDA-MB-231 cell model in vitro and a MDA-MB-231 xenograft model in vivo.

Results: The prepared RGD-modified docetaxel-loaded lipid-core micelles were spherical with a particle size of $16.44 \pm 1.35 \mathrm{~nm}$, zeta potential of $-19.24 \pm 1.24 \mathrm{mV}$, and an encapsulation efficiency of $96.52 \pm 0.43 \%$. The drug delivery system showed sustained release properties and could significantly enhance docetaxel uptake by MDA-MB-231 tumor cells in vitro, which was proved to be a caveolae pathway mediated process requiring ATP, Golgi apparatus, and acid lysosomes. The results of the pharmacokinetic study displayed that the area under the curve of the targeted micelles was 3.2-times higher than that of docetaxel commercial injections. Furthermore, in a MDA-MB-231 tumor-bearing mice model, a higher antitumor efficacy than docetaxel commercial injections was displayed, and the safety experiments showed that the micellar material did not cause major organ damage after intravenous administration in mice.

Conclusion: The novel RGD-modified PEGylated lipid-core micelle delivery system significantly improved the antitumor effects and reduced the side-effects of docetaxel, providing a promising therapeutics for the treatment of TNBC.

Keywords: docetaxel, lipid-core micelles, RGD peptide, breast cancer therapy

\section{Introduction}

Breast cancer is a significant cause of cancer-related deaths in females. ${ }^{1}$ It is a common malignant tumor whose occurrence is closely related to differentiation disorder, uncontrolled proliferation, and apoptosis of cells. At present, the clinical treatments for breast cancer mainly include chemotherapy, hormone therapy, surgery, and radiation. ${ }^{2}$ Although remarkable progress of these treatments has significantly improved the disease control rate of certain breast cancer, there are still no effective treatments for triple negative breast cancer (TNBC), which accounts for approximately $15 \%$ of all breast cancer cases. Due to its high tendency toward metastasis and the lack of effective targeted therapy and endocrine therapy drugs, the 5 -year survival rate of TNBC patients is less than $20 \%{ }^{3}$ 
Docetaxel (DTX) is a kind of compound synthesized by the structural modification of paclitaxel extracted from the needles of the berry yew. ${ }^{4}$ Its antitumor mechanism is the same as that of paclitaxel, with about twice the activity of paclitaxel. It can inhibit mitosis and cell proliferation by forming nonfunctional microtubule bundles. ${ }^{5}$ It is one of the most effective single dose chemotherapy drugs for metastatic breast cancer and non-small cell lung cancer, ${ }^{6}$ and it is also one of the drugs used in the standard chemotherapy regimen for triple negative breast cancer. ${ }^{7}$ Because DTX is highly lipophilic and insoluble in water, Tween 80 and ethanol are often used with it for solubilization in clinical application. These solubilizers have serious side-effects, causing most patients to have severe allergic reactions, like cumulative fluid retention, hypersensitivity reactions, and nausea. ${ }^{8}$ It has been reported that the commercial injections of DTX (Taxotere ${ }^{\circledR}$ ) cause serious sideeffects due to DTX itself or polysorbate $80 .{ }^{9}$ Therefore, the studies of nano-formulations are growing for improving the therapeutic effect of DTX and overcoming its disadvantages.

Combining active targeting and passive targeting could greatly improve the therapeutic effects on triple negative breast cancer. ${ }^{10,11}$ However, there are still many problems in the active targeted drug delivery system, such as low encapsulation rate and poor stability, which lead to the early release of drugs in vivo and fast elimination in vivo, greatly limiting their production and marketing. ${ }^{12-}$ ${ }^{14}$ The lipid core micelles are assembled by water-soluble amphiphilic surfactants, which take the hydrophilic end of amphiphilic polymers as the shell and the hydrophobic end as the core. Compared with other drug delivery systems, lipid core micelles have many advantages, such as strong hydrophobic effect which contributes to high drug loading for lipophilic drugs, ${ }^{15}$ and small particle size which can make drugs accumulate in tumor tissue through passive targeting. Besides, low critical micelle concentration endows them with good dilution stability after in vivo administration. ${ }^{16}$ After 48 hours of interaction with plasma, they can still maintain their particle size stability.${ }^{17}$ What's more, the preparation process is much easier, enabling large scale production of these formulations. ${ }^{18}$

In recent years, the overexpression on tumor and tumor vascular systems of extracellular matrix binding integrin receptors (such as $\alpha_{v} \beta_{3}$ and $\alpha_{v} \beta_{5}$ ) draws more and more attention in tumor targeting delivery. ${ }^{19}$ Song et $\mathrm{al}^{20}$ designed dual-targeting nanovesicles which could combine with $\alpha_{v} \beta_{3}$ integrin to enhance specificity to dynamic tumor cells in vitro and in vivo. The expression of $\alpha_{\mathrm{V}} \beta_{3}$ in breast cancer cells is higher than that in normal cells, especially in metastatic breast tumors. ${ }^{21}$ Besides, researches have proved that the expression of $\alpha_{v} \beta_{3}$ receptors on the surface of TNBC cells is much higher than other non-TNBC cells. $^{22,23}$ TNBC cells such as MDA-MB-231 cells could overexpress $\alpha_{v} \beta_{3}$ integrin. ${ }^{24}$ Therefore, $\alpha_{v} \beta_{3}$ integrin has become a new target for TNBC treatment. RGD peptide is a kind of short peptide comprising arginine-glycine-aspartic acid (Arg-Gly-ASP), which can mediate the interaction between cells and cells, or the extracellular matrix and cells, and recognize the $\alpha_{v} \beta_{3}$ receptor expressed on the surface of tumor cells and tumor blood vessels. ${ }^{25}$ In addition, a RGD peptide modified nano-delivery system showed good tumor targeting ability in vitro $^{26}$ and in vivo. $^{22,27}$ Therefore, RGD peptide is suitable to be a targeting molecule for TNBC treatment.

In this study, using RGD peptide as a targeting molecule, and amphiphilic polymer PEG-DSPE as a carrier, the RGD peptide mediated TNBC targeted DTX loaded PEGDSPE lipid-core micelles (RGD-DTX-M) were prepared. The micelle shell is composed of hydrophilic PEG to form a hydration layer, which could limit the phagocytosis of the reticuloendothelial system and prolong the circulation time of micelles in vivo. After RGD modification, the micelles exhibit active targeting which could bind to the $\alpha_{v} \beta_{3}$ integrin receptor, realizing the targeted transportation of DTX to the TNBC cells, and at the same time improving the DTX curative effect and reducing its side-effects. Their physicochemical properties, in vitro release behavior, pharmacokinetics, tumor targeting ability, targeting mechanism, and toxicity were studied. This new TNBC targeted delivery system is expected to increase the drug loading of DTX, prolong the drug retention time in the blood, and improve the treatment effect on triple negative breast cancer, having a high clinical application prospect.

\section{Materials and Methods \\ Materials}

DTX was bought from Shanghai Techwell Biopharmaceutical Co., Ltd (Shanghai, China). DTX commercial injections were supplied by the Shanghai Acebright Pharmaceuticals Group Co., Ltd. (Shanghai, China). Cyclo (Arg-Gly-Asp-DPhe-Cys) (RGD) was purchased from Chinese Peptide (Hangzhou, China, purity $>98 \%$ ). 1,2-distearoyl-sn-glycero-3-phosphoethanolamineN- [(polyethylene glycol)-2000] (DPSE-PEG2000) was 
purchased from Nanosoft Polymers (NC, USA). 1,2-distearoyl-sn-glycero-3-phosphoethanolamine- $\mathrm{N}$-[maleimide (polyethylene glycol)-2000] (DSPE-PEG2000-Maleimide) was purchased from Avanti Polar Lipids (Alabaster, AL, USA). Tween 80, acetonitrile, chloroform, and methanol were purchased from Sinopharm Chemical Reagent Co., Ltd (Shanghai, China). Filipin, sodium azide, chlorpromazine, brefeldin A (BFA), colchicine, and monensin were supplied by Sigma-Aldrich Co (MO, USA). Hank's balanced salt (HBS) and phosphate buffer saline (PBS) were purchased from Shanghai Basal Media Technologies Co., Ltd (Shanghai, China). Dulbecco's modified Eagle medium (DMEM) and fetal bovine serum (FBS) were purchased from Thermo Fisher Technology (China) Co., Ltd. Cell Counting Kit-8 (CCK-8) was purchased from Dojindo Laboratories (Kumamoto, Japan).

\section{Cell Lines and Animals}

A human breast cancer cell line (MDA-MB-231) that was provided by the Type Culture Collection of the Chinese Academy of Sciences (Shanghai, China), was grown in DMEM containing $10 \%$ FBS in a $37^{\circ} \mathrm{C}$ incubator with $5 \%$ $\mathrm{CO}_{2}$. Cell proliferation and toxicity assay were performed by using CCK-8.

Female Sprague Dawley rats of $200 \pm 20 \mathrm{~g}$ and female nude mice aged 4-6 weeks were supplied by Shanghai Super B\&K Laboratory Animal Corporation Ltd (Shanghai, China). Prior to experiments, the animals were raised at the temperature of $22^{\circ} \mathrm{C} \pm 2^{\circ} \mathrm{C}$ under natural light/dark conditions with access to food and water freely for a week. The animals used for the experiment were treated following the approval of all the protocols that were set by the ethical committee of Shanghai University of Traditional Chinese Medicine (Shanghai, China). The in vivo experiments were also approved by the ethical committee of Shanghai University of Traditional Chinese Medicine. All experiments follow the ethical principles of experimental animal welfare and make every effort to minimize suffering.

\section{Preparation of RGD-DTX-M}

In brief, $2 \mathrm{mg}$ DSPE-PEG2000-Maleimide and $80 \mathrm{mg}$ DSPE-PEG2000 were dissolved in $8 \mathrm{~mL}$ chloroform. Then, the solution was mixed up with $2.05 \mathrm{mg}$ DTX which was dispersed in $2 \mathrm{~mL}$ methanol. The mixture was dried under reduced pressure at $38^{\circ} \mathrm{C}$ until an arid thinfilm formed. After being maintained in a vacuum freezedrying machine for 24 hours to remove residual solvents, 8
$\mathrm{mL}$ HBS was added for hydration. Subsequently, the solution was filtrated through $0.22 \mu \mathrm{m}$ filter membrane. Finally, the micelle solution was stirred for 12 hours with $1 \mathrm{mg} / \mathrm{mL}$ RGD which was dissolved in HBS. The blank micelles (BMs) and RGD-free micelles (DTX-M) were also prepared following the same described procedures, except for adding DTX or RGD. Before in vivo and in vitro experiments, the free RGD in the micelles was removed by ultrafiltration with centrifugal filters $(3 \mathrm{kDa}$, Millipore).

\section{Characterization of Micelles}

Size and zeta potential measurements of micelles including BMs, DTX-M, and RGD-DTX-M were performed at room temperature through a dynamic light scattering (DLS) method using a Nicomp Particle Sizing system (380ZLS, Santa Barbara, CA, USA). After the samples were diluted by distilled water and negatively stained with phosphotungstic acid, the morphology of micelles was observed by transmission electron microscope (TEM, H-6009IV, Hitachi, Japan). Drug loading (DL) and encapsulation efficiency (EE) of RGD-DTX-M were determined by high-performance liquid chromatography (HPLC) on a Kromasil 100-5C18 column (5 $\mu \mathrm{m}$, $250 \times 4.6 \mathrm{~mm})$ at $30^{\circ} \mathrm{C}$. The mobile phase was prepared with a mixture of acetonitrile and ultrapure water (55:45, $\mathrm{v} / \mathrm{v})$. The DTX was detected at the wavelength of $230 \mathrm{~nm}$ (flow rate: $1.0 \mathrm{~mL} / \mathrm{min}$ ). The selectivity, linearity, precision, and recovery of this method were fully validated. EE and DL were determined using Eqns. (1) and (2), respectively.

EE $(\%)=$ Weight of the drug encapsulated in micelles $(\mathrm{mg}) /$ Weight of the total drug added $(\mathrm{mg}) \times 100 \%(1)$

DL $(\%)=$ Weight of the drug encapsulated in micelles $(\mathrm{mg}) /$ Weight of the total micelles $(\mathrm{mg}) \times 100 \%(2)$

\section{In vitro Release of DTX from Micelles}

The release kinetics of DTX-M and RGD-DTX-M were tested through the dialysis method (PBS with $0.1 \%$ Tween-80 with or without plasma as the release medium). Briefly, dialysis membrane bags filled with $2 \mathrm{~mL}$ preformed micelles were transferred into $50 \mathrm{~mL}$ centrifuge tubes full of release medium. The whole device was then immersed in a $37^{\circ} \mathrm{C}$ water bath gently shaken at $100 \mathrm{rpm}$ for 24 hours. At the pre-designed time points, the release medium was extracted and replaced with fresh buffer solution. The concentrations of DTX in release medium were determined by the HPLC method described above. 
In vitro Uptake of RGD-DTX-M by MDAMB-23 I Cells

The triple-negative human breast cancer cell line MDAMB-231 was used to evaluate the uptake of RGD-DTX-M and DTX-M by triple-negative breast cancer cells. The MDA-MB-231 cells were inoculated into 24 well plates and cultured to a density at about $3 \times 10^{5}$ cells per well. Then RGD-DTX-M and DTX-M were administrated respectively. After that, cold PBS was added to stop the uptake at predetermined time points, and $0.05 \%$ SDS was added to lyse the cells. The cell lysate was taken to determine the protein concentration by a BCA kit and the concentration of DTX by HPLC. The uptake index (UI) was determined using the following formulae:

\section{UI $(\mu \mathrm{g} / \mathrm{mg})=$ Concentration of DTX $(\mu \mathrm{g} / \mathrm{mL}) /$ Concentration of protein $(\mathrm{mg} / \mathrm{mL})$}

\section{Uptake Mechanism of RGD-DTX-M by MDA-MB-23। Cells}

The MDA-MB-231 cells were inoculated into 24 well plates and cultured to a density at about $3 \times 10^{5}$ cells per well. After different inhibitors $(2.6 \mathrm{mg} / \mathrm{mL}$ sodium azide, $10 \mu \mathrm{g} / \mathrm{mL}$ filipin, $20 \mu \mathrm{g} / \mathrm{mL}$ chlorpromazine, $4 \mu \mathrm{g} / \mathrm{mL}$ colchicine and $20 \mu \mathrm{g} / \mathrm{mL}$ BFA, $140 \mathrm{ng} / \mathrm{mL}$ monensin) were added, respectively, the cells were placed in a $37^{\circ} \mathrm{C}$ incubator for 30 minutes. Then, the cells were rinsed with PBS and incubated in medium containing RGD-DTX-M or DTX-M for 1 hour. After absorbing and discarding the culture solution in the culture plate, $0.05 \%$ SDS was used to lyse the cells. The cell lysate was taken to determine the protein concentration and the concentration of DTX. The UIs were calculated and compared between different groups.

\section{Pharmacokinetic Studies of RGD-DTX-M} The pharmacokinetics of the RGD-DTX-M and a DTX commercial injection (Shanghai Acebright Pharmaceuticals Group Co., Ltd., lot no. 9021191204) were investigated in healthy female SD rats following intravenous administration. The rats were randomly assigned into two groups (four rats per group), and administered via tail vein at a dose equivalent to $2 \mathrm{mg} / \mathrm{kg}$ DTX. The blood samples (approximately $0.5 \mathrm{~mL}$ ) were collected from the orbital cavity into the micro centrifuge tubes containing heparin at $0,0.083,0.25,0.5,1,2,4,8$, and 12 hours. After the plasma was separated from the obtained blood by centrifuging the blood samples at $8,000 \mathrm{rpm}$ for 3 minutes at $4^{\circ} \mathrm{C}$, the plasma samples were frozen at $-80^{\circ} \mathrm{C}$ until analysis.

The pharmacokinetic data was analyzed using a noncompartmental model by the Drug and Statistics (DAS) software (version 2.1.1, Mathematical Pharmacology Professional Committee, China).

\section{Biodistribution of Micelles in MDA-MB- 23I Tumor-Bearing Mice}

The female nude mice (6-8 weeks of age and 18-20 g) were subcutaneously inoculated with $2 \times 10^{6}$ MDA-MB231 breast cancer cells for constructing the orthotopic tumor-bearing mice model. In order to evaluate the biodistribution of the RGD-modified micelles in vivo, the near-infrared fluorescence dye DiR was used to replace DTX as the fluorescence probe to be encapsulated in the micelles. The preparation methods of DiR loaded micelles (RGD-DiR-M and DiR-M) are according to the preparation of RGD-DTX-M and DTX-M, leading to similar particle size (around $20 \mathrm{~nm}$ ) and DiR content $(125 \mu \mathrm{g} /$ $\mathrm{mL}$ ) of RGD-DiR-M and DiR-M. Mice were injected intravenously with $0.2 \mathrm{~mL}$ of free DiR, DiR-M, and RGD-DiR-M, respectively (the injected dose of DiR in each mouse was $2 \mathrm{mg} / \mathrm{kg}, \mathrm{n}=4$ ). At 4,8 , or 24 hours post-injection, the mice were scanned using a near-infrared fluorescence imaging system (IVIS Lumina XR, PerkinElmer, USA; ex. $748 \mathrm{~nm}$, em. $780 \mathrm{~nm}$ ) to acquire in vivo images. At 24 hours post injection, the main organs and the tumors were dissected from the sacrificed orthotopic tumor models for ex vivo imaging, and the biological distribution of the micelles was evaluated.

\section{Antitumor Effect of RGD-DTX-M on MDA-MB-23 I Tumor-Bearing Nude Mice}

Female nude mice (4-6 weeks old) were selected to establish MDA-MB-231 breast cancer xenograft models for investigating the antitumor effect of RGD-DTX-M. $2 \times 10^{6}$ MDA-MB-231 cells suspended in $0.1 \mathrm{~mL}$ PBS were subcutaneously injected at the right flank of each nude mouse. After 10 days of implantation, the mice with a tumor volume ranging from $90-110 \mathrm{~mm}^{3}$ were randomly divided into five groups (normal saline, DTX commercial injection, RGD-M, DTX-M, and RGD-DTXM) $(n=5)$. And different formulations were administrated intravenously via the tail vein every 4 days for four times at a dose equal to $5 \mathrm{mg} / \mathrm{kg}$ DTX. During the study, the 
tumor volume and body weight of the mice were measured every day. For calculating the tumor volume, it was based on the equation $\left(a \times b^{2}\right) / 2$, where $a$ and $b$ were the length of the the cuboid and the width of the cuboid, respectively. On the 28th day, all the alive nude mice were sacrificed and the tumors were collected and weighed.

\section{Preliminary Safety Studies in vitro and in vivo}

MDA-MB-231 cells were used to evaluate the safety of the blank micelle RGD-M. Briefly, approximately $5 \times 10^{4}$ cells were seeded per well into a 96 -well plate, and placed in a $5 \%$ $\mathrm{CO}_{2}$ incubator at $37^{\circ} \mathrm{C}$. After incubating for 24 hours, the cells reaching $80 \%$ confluence were washed twice with PBS and treated with varying concentrations of blank micelles with or without RGD modification at $37^{\circ} \mathrm{C}$ for 24 hours. The cell viability was then measured by MTT assay.

After the antitumor-effect experiment, the nude mice were killed on the 28th day, and heart, lungs, liver, spleen, and kidneys were taken out and preserved for tissue slice to assess systemic toxicity of RGD-DTX-M in vivo through hematoxylin and eosin (H\&E) staining.

\section{Statistical Analysis}

Data are expressed as the mean \pm standard deviation (SD). Statistical evaluation was analyzed by a two-tailed Student's $t$-test or one-way ANOVA analysis of variance with Prism 7.0 Software when comparing two groups or larger than two groups, respectively. A $p$-value $<0.05$ was considered to be statistically significant.

\section{Results \\ Characterization of DTX-M and RGD- DTX-M}

Taking particle size, zeta potential, encapsulation efficiency, and drug loading as indicators, the changes of DTX-M before and after RGD modification were investigated. The results are shown in Figure 1E, indicating that there is no significant difference between DTX-M and RGD-DTX-M in physicochemical properties. With high encapsulation efficiency, the two kinds of micelles were found to be in the size range of 14-20 nm, displaying a strong negative zeta potential, which is conducive to the micelle stability against fusion and aggregation. The TEM photographs of DTX-M and RGD-DTX-M are shown in Figures 1A and B. The DTX-M and RGDDTX-M were spherical in shape, and were uniformly distributed. The in vitro release results showed that DTX-M was released completely in PBS buffer in about 4 hours, while plasma slowed down the release speed (Figures 1C and D). In PBS containing plasma, both of DTX-M and RGD-DTX-M totally released in 8 hours, showing sustained-release effects (Figures 1C and D).

\section{In vitro Uptake of RGD-DTX-M by MDA- MB-23 I Cells}

The influences of incubation time and concentration of micelles on the cell uptake of RGD-DTX-M and DTX-M were investigated. As shown in Figure 2A, the uptake of the formulations increased with time, and, after 2 hours, the uptake of RGD-DTX-M was significantly higher than that of DTX-M, and the uptake of both the two kinds of formulations was significantly higher than that of DTX solution. Besides, the cell uptake of different preparations rose with the increase of formulation concentration, and the uptake of RGD-DTX-M was also higher than that of DTX$\mathrm{M}$ at the same formulation concentration (Figure 2B).

\section{Uptake Mechanism of RGD-DTX-M by MDA-MB-23। Cells}

In order to elucidate the cell entry pathways of RGDDTX-M, the endocytosis mechanism of micelles was investigated. As shown in Figure 2C, after treating with sodium azide, brefeldin A (BFA) and monensin for 30 minutes, the uptake indexes of micelles by MDA-MB231 cells were significantly reduced, illustrating that the endocytosis of RGD-DTX-M might be a caveolin mediated process which needs ATP, and involves Golgi body and acid lysosomes. Furthermore, although both the group of RGD-DTX-M and the group of DTX-M showed the same inhibition trend after treating with different kinds of inhibitors, the inhibition rate between the two groups was different. For example, after cells were treated with sodium azide, the inhibition rate of uptake index of DTX-M was approximately 3-times that of RGD-DTX-M, which demonstrated a more pronounced inhibition of uptake of RGD-DTX-M.

\section{Pharmacokinetics of RGD-DTX-M}

The plasma concentration-time curves of DTX after i.v. administration of DTX commercial injections and RGDDTX-M in rats are demonstrated in Figure 3. The calculated pharmacokinetic parameters are shown in Table 1. The area 

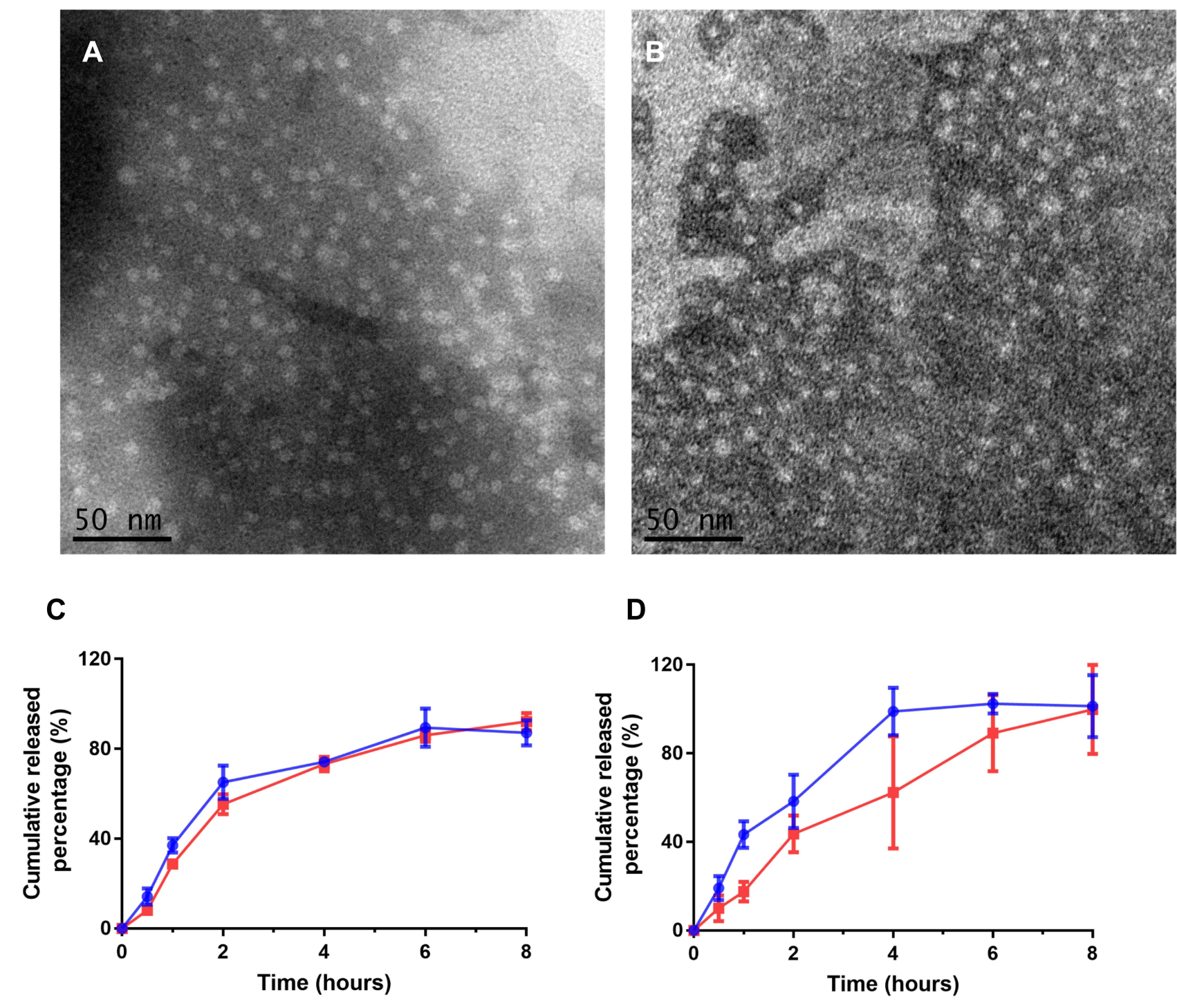

D

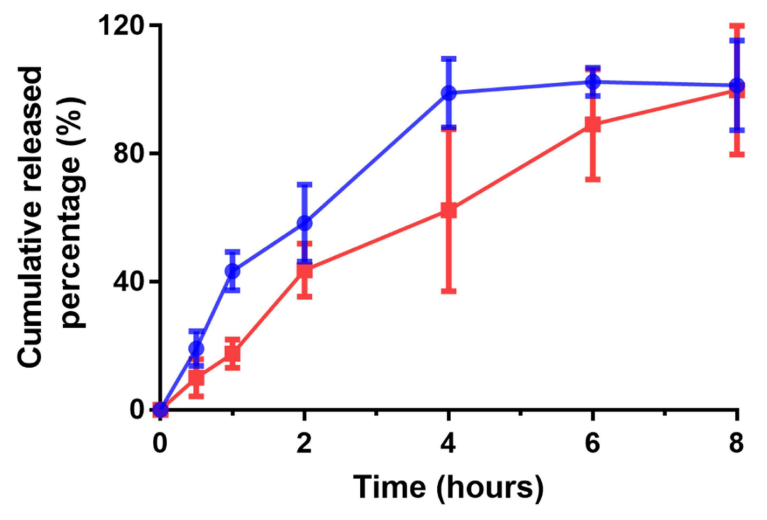

In PBS

In PBS containing serum

E

\begin{tabular}{ccccc}
\hline Formulations & $\begin{array}{c}\text { Particle } \\
\text { Size } \\
(\mathbf{n m})\end{array}$ & $\begin{array}{c}\text { Zeta potential } \\
(\mathbf{m V})\end{array}$ & $\begin{array}{c}\text { Encapsulation } \\
\text { Efficiency } \\
(\%)\end{array}$ & $\begin{array}{c}\text { Drug Loading } \\
(\%)\end{array}$ \\
\hline DTX-M & $16.55 \pm 1.96$ & $-17.20 \pm 6.46$ & $96.76 \pm 2.32$ & $2.34 \pm 0.05$ \\
RGD-DTX-M & $16.44 \pm 1.35$ & $-19.24 \pm 1.24$ & $96.52 \pm 0.43$ & $2.33 \pm 0.01$ \\
\hline
\end{tabular}

Figure I Characterization of micelles. TEM photos of RGD-DTX-M (A) and DTX-M (B). Drug release profiles of RGD-DTX-M (C) and DTX-M (D) in PBS with or without serum at the temperature of $37^{\circ} \mathrm{C} \pm 0.5^{\circ} \mathrm{C}$. (E) Characteristics of DTX-M and RGD-DTX-M $(n=3)$. 
A

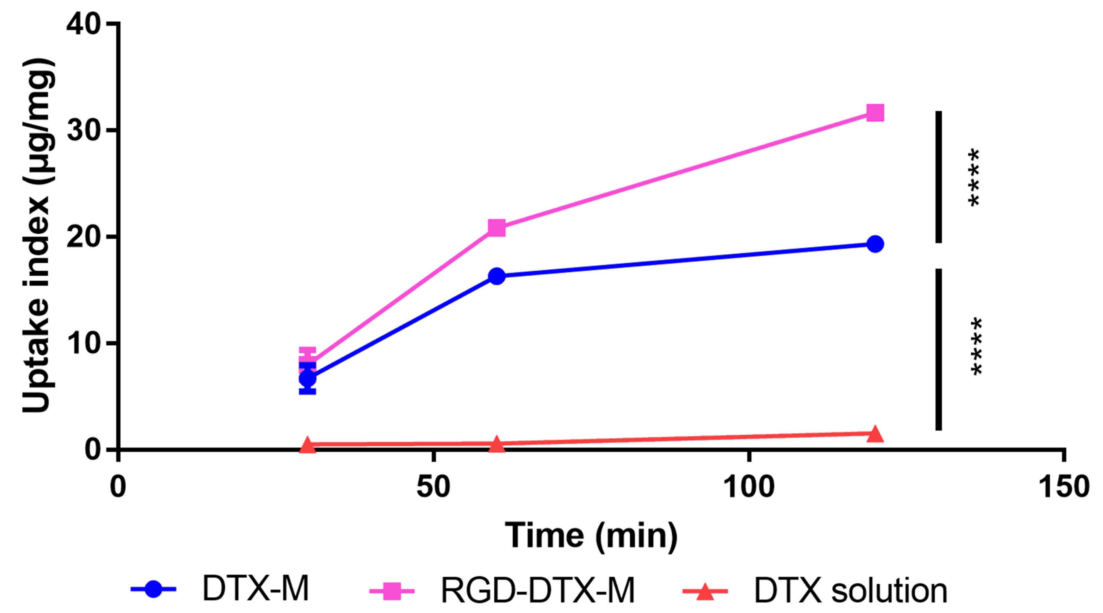

B

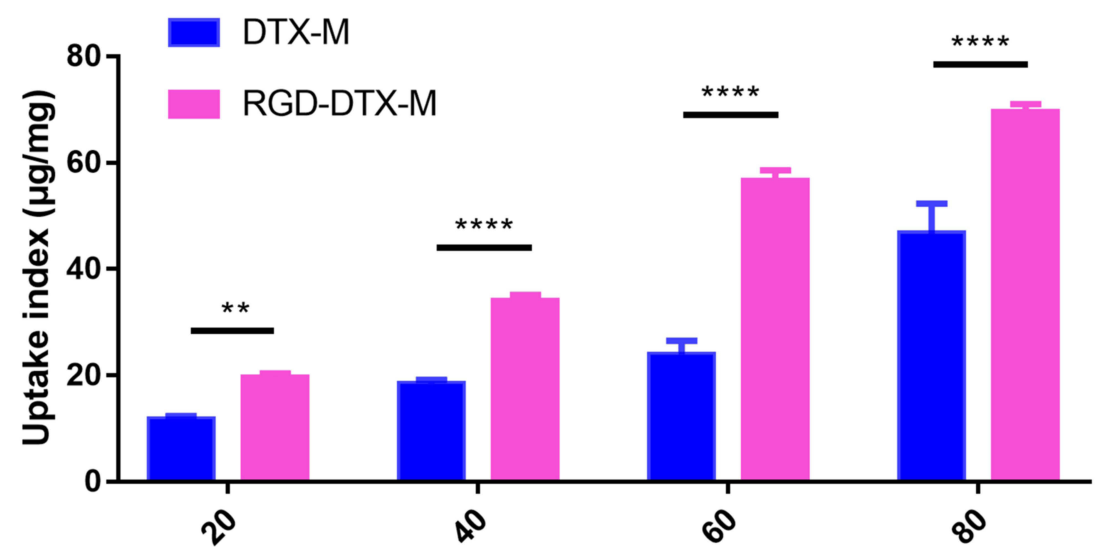

Formulation concentration

$(\mu \mathrm{g} / \mathrm{mL})$

C

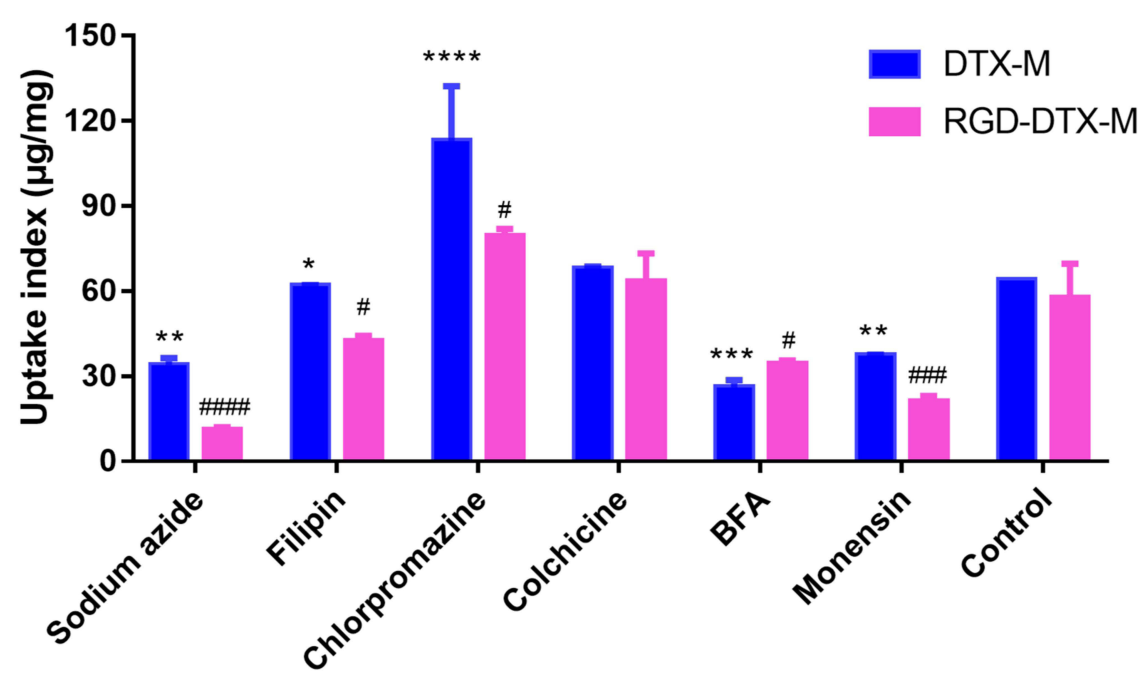

Figure 2 In vitro uptake of RGD-DTX-M by MDA-MB-23I cells. Effect of incubation time (A) and formulation concentration (B) on micelle uptake by MDA-MB-23I cells $(\mathrm{n}=3, * * p<0.01, * * * * p<0.000 \mathrm{I})$. Uptake mechanism (C) of RGD-DTX-M by MDA-MB-23I cells $(\mathrm{n}=3, * p<0.05, * * p<0.01, * * * p<0.001$, $* * * * p<0.000 \mathrm{I}$, DTX-M compared with

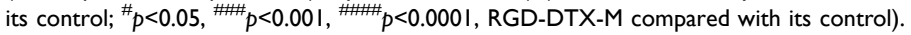




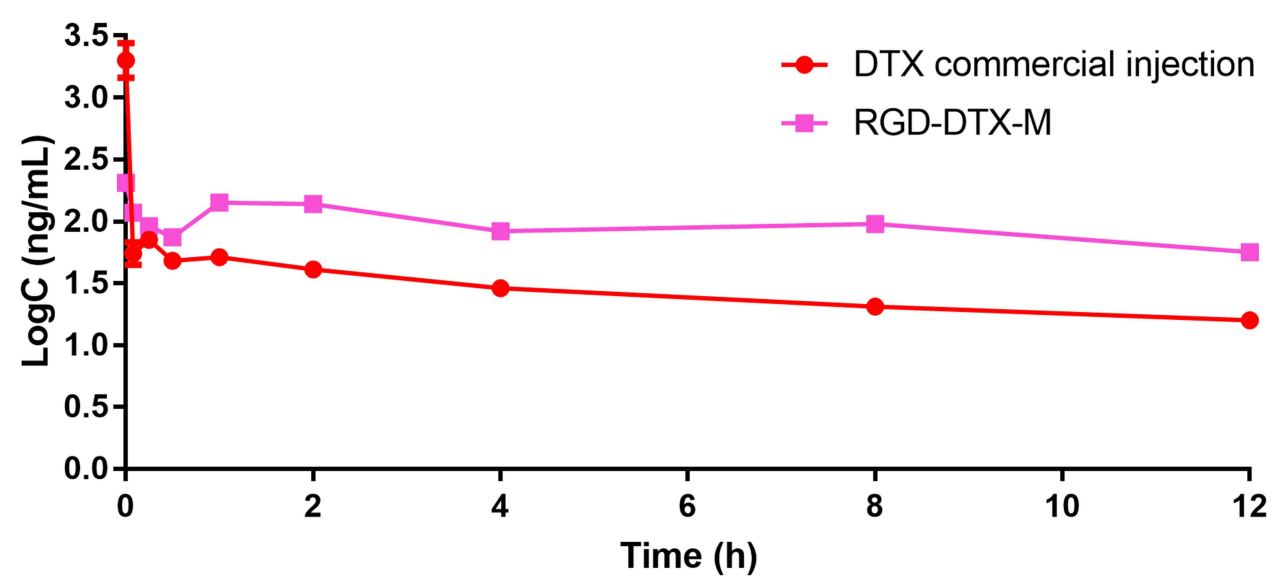

Figure 3 The concentration-time curves of DTX in plasma followed by i.v. injection of DTX commercial injection and RGD-DTX-M in rats at a DTX dose of 2 mg kg $^{-1}$ $(n=4)$.

Abbreviation: C, plasma concentration of DTX.

under the curve (AUC) was significantly increased from $557.55 \pm 14.13 \mu \mathrm{g} / \mathrm{L} \cdot \mathrm{h}$ for the commercial injection of DTX to $1771.65 \pm 63.03 \mu \mathrm{g} / \mathrm{L} \cdot \mathrm{h}$ for RGD-DTX-M, which corresponds to a significant reduction of the total body clearance (TBC) from $3.59 \pm 0.09 \mathrm{~L} / \mathrm{kg} \cdot \mathrm{h}$ to $1.07 \pm 0.12 \mathrm{~L} / \mathrm{kg} \cdot \mathrm{h}$. Furthermore, $\mathrm{t}_{1 / 2}$ for the commercial injection of DTX was $5.57 \pm 0.51 \mathrm{~h}$, which is statistically shorter than the 13.25 $\pm 2.54 \mathrm{~h}$ for RGD-DTX-M. These results demonstrated better sustained-release properties of the RGD-DTX-M delivery system compared with the commercial preparations.

\section{Biodistribution of Micelles in MDA-MB- 23I Tumor-Bearing Mice}

The in vivo targeting ability of RGD-DTX-M micelles was investigated on MDA-MB-231 tumor-bearing nude mice. A near-infrared fluorescence dye DIR was used as the fluorescent chromogenic agent entrapped in micelles for studying the biodistribution of micelles by a fluorescence

Table I Pharmacokinetic Parameters of DTX in Plasma Followed Intravenous Administration of DTX Commercial Injection and RGD-DTX-M

\begin{tabular}{|l|c|c|}
\hline PK Parameters & $\begin{array}{c}\text { DTX Commercial } \\
\text { Injection }\end{array}$ & RGD-DTX-M \\
\hline $\mathrm{t}_{\mathrm{I} / 2}(\mathrm{~h})$ & $5.57 \pm 0.5 \mathrm{I}$ & $13.25 \pm 2.54 * *$ \\
$\mathrm{C}_{\max }(\mu \mathrm{g} / \mathrm{L})$ & $2014.95 \pm 637.67$ & $204.46 \pm 6.29 * *$ \\
$\mathrm{t}_{\max }(\mu \mathrm{g} / \mathrm{L})$ & 0 & 0 \\
$\mathrm{AUC}$ & $557.55 \pm 14.13$ & $1771.65 \pm 63.03 * * * *$ \\
$\mathrm{TBC}(\mathrm{L} / \mathrm{kg} / \mathrm{h})$ & $3.59 \pm 0.09$ & $1.07 \pm 0.12 * * * *$ \\
\hline
\end{tabular}

Abbreviations: AUC, area under the curve; TBC, total body clearance; $\mathrm{t}_{1 / 2}$, drug half-life; $\mathrm{C}_{\max }$, maximum concentration. imaging system. As shown in Figure 4A, compared with the other two groups, the tumor fluorescence signals of RGD-DiR-M treated mice were significantly stronger at 4 24 hours. As the time was prolonged, RGD-DiR-M began to concentrate in the tumor, and the tumor accumulation of fluorescent signals peaked at approximately 8 hours. The ex vivo images at 24 hours post-injection displayed that the fluorescence intensity of RGD-DiR-M was stronger in tumors and weaker in other organs than the DiR-M (Figure 4B-D). These results suggested that RGD-DTX-M might serve as a better DTX delivery system because of its better tumor targeting capacity in vivo.

\section{Antitumor Effect of RGD-DTX-M on MDA-MB-23I Tumor-Bearing Nude Mice}

To verify the antitumor activity of RGD-DTX-M, nude mice bearing MDA-MB-231 human breast cancer cells were treated with DTX commercial injections, DTX-M and RGD-DTX-M at the DTX dose of $5 \mathrm{mg} / \mathrm{kg}$. The antitumor effects of DTX-M and RGD-DTX-M on MDA-MB-231 bearing nude mice are illustrated in Figure 5, compared with normal saline (control group), RGD-M (the same dose of vehicle as RGD-DTX-M at 5 $\mathrm{mg} / \mathrm{kg}$ DTX), and DTX commercial injection. Normal saline and RGD-M vehicle treatments had no significant effect on the tumor growth, with the tumor volumes increasing rapidly. The treatment with DTX and DTX$M$ was effective in tumor regression to some extent, but neither of them had advantages when they were compared to the RGD-DTX-M, which was more efficacious to suppress tumor growth (Figures 5A, C, E, and 4D). As 
A

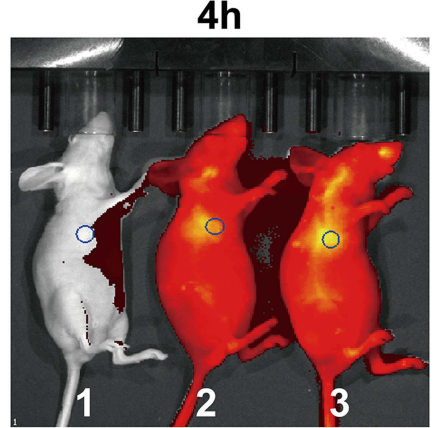

B

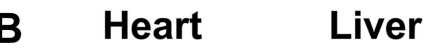

$8 h$

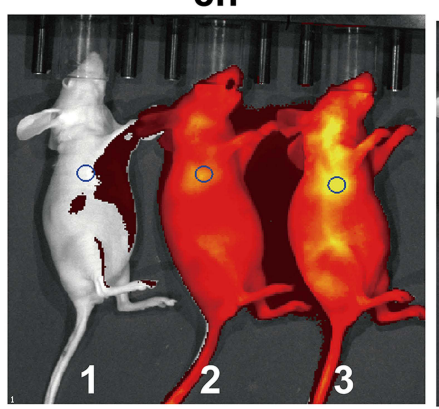

Spleen Lung
$24 h$

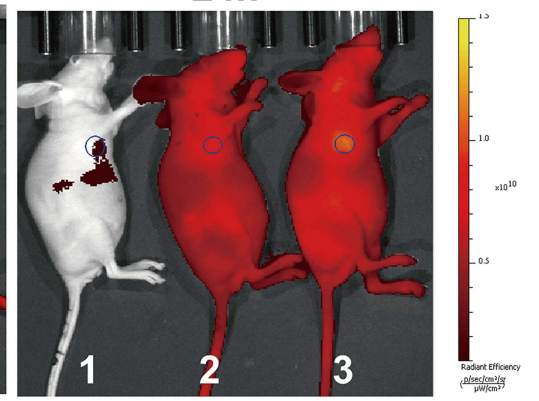

Kidney Tumor

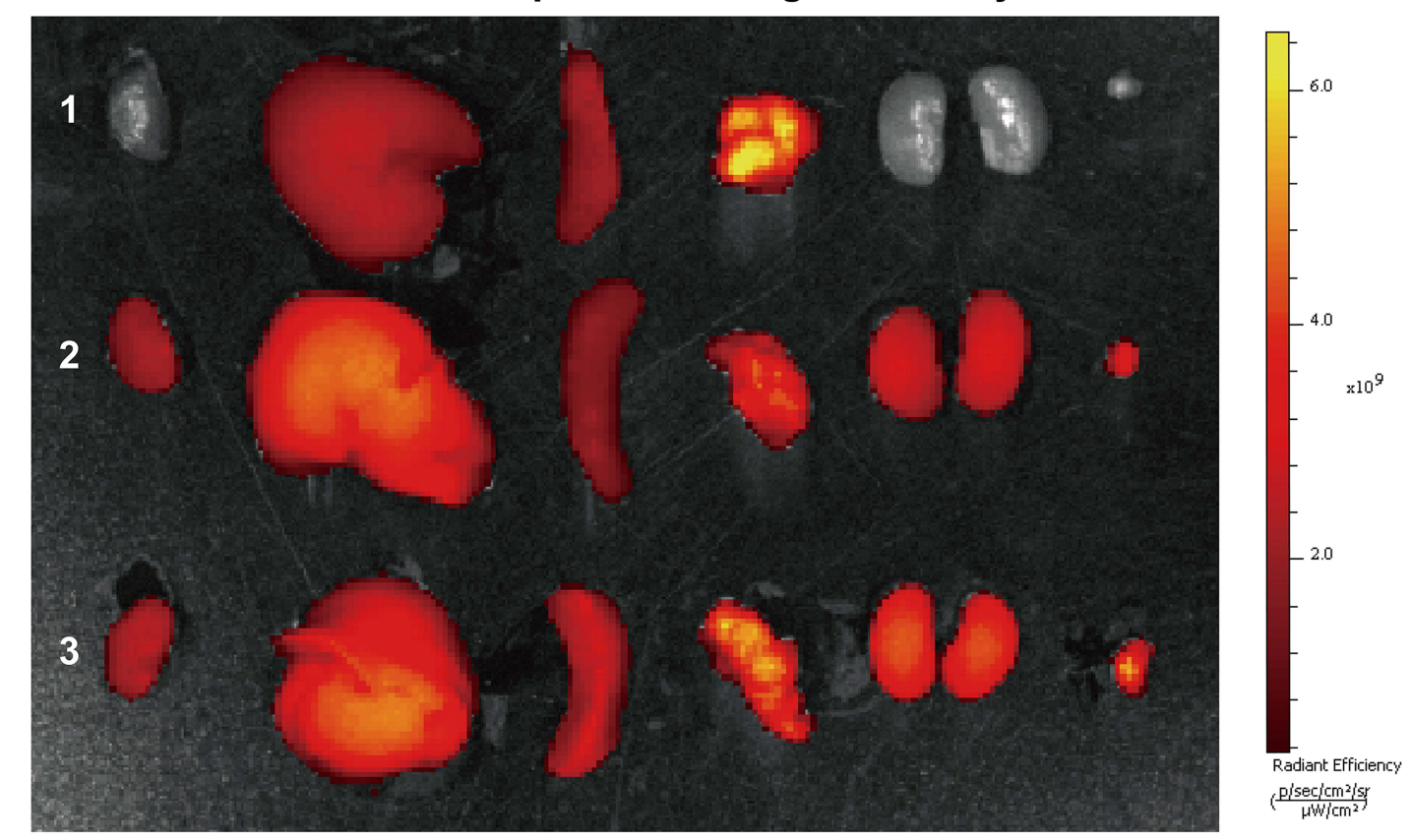

C

D
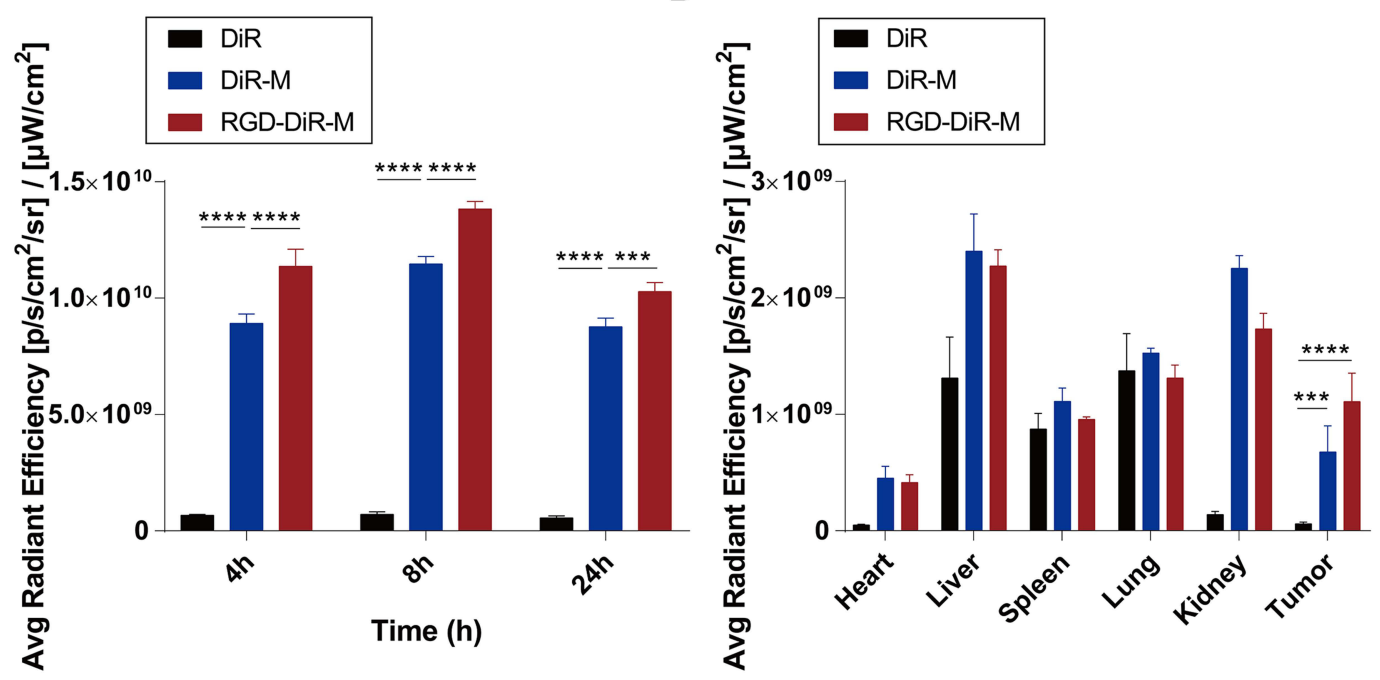

Figure 4 In vivo biodistribution of free DiR (I), DiR-M (2) and RGD-DiR-M (3). In vivo fluorescence images of the tumor-bearing mice after intravenous injection at 4, 8, and 24 hours post injection (A); fluorescence images of ex vivo main organs and tumors at 24 hours post injection (B); the quantitative analysis of fluorescence intensity in tumors at 4,8 , and 24 hours post injection $(\mathbf{C})$; the quantitative analysis of fluorescence intensity in ex vivo mean organs and tumors $(\mathbf{D})(\mathrm{n}=4$, $* * * p<0.00 \mathrm{I}$, $* * * * p<0.000 \mathrm{I})$. 
A

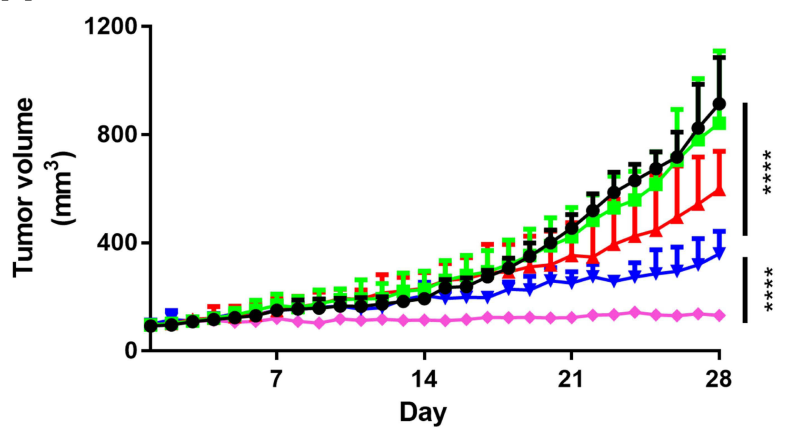

C

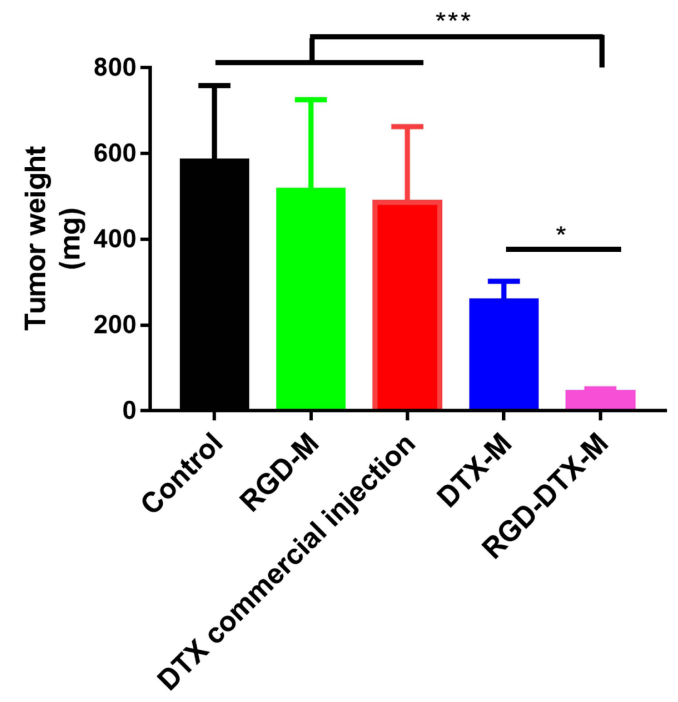

E
B

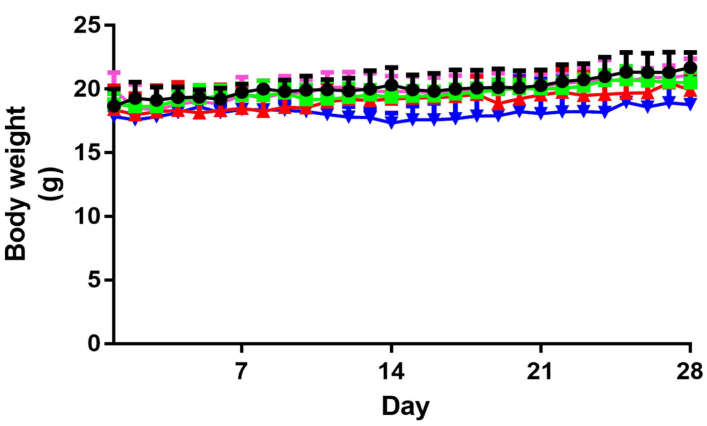

$\rightarrow$ DTX-M $\rightarrow$ RGD-DTX-M

D

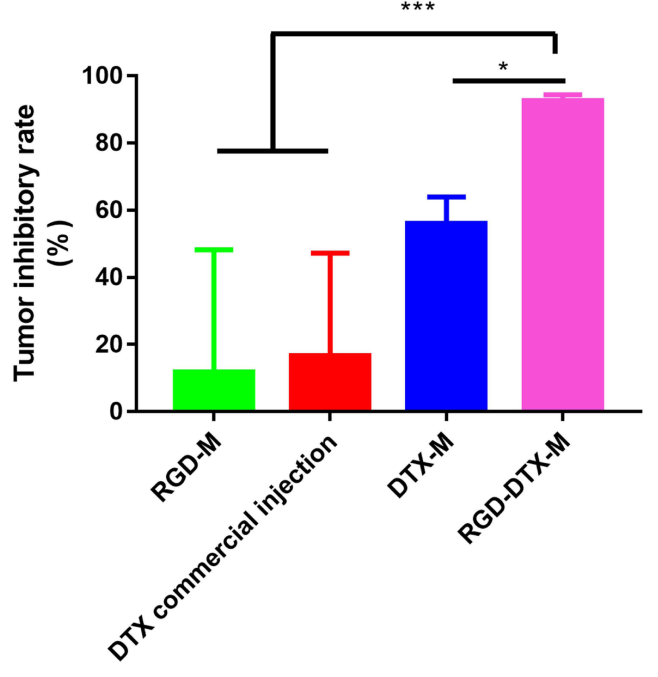

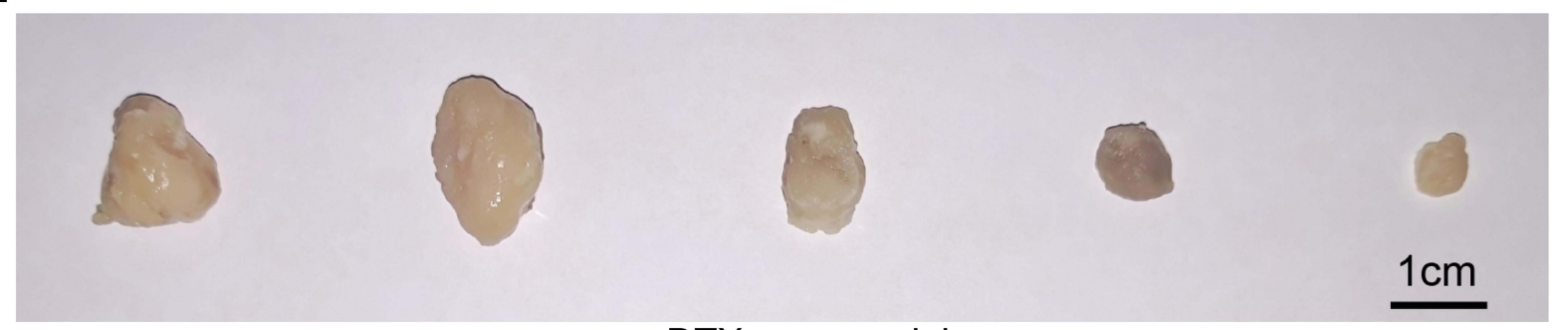

F Control

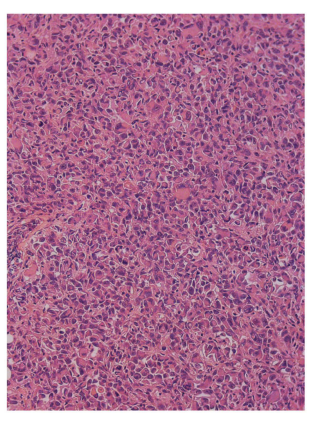

RGD-M

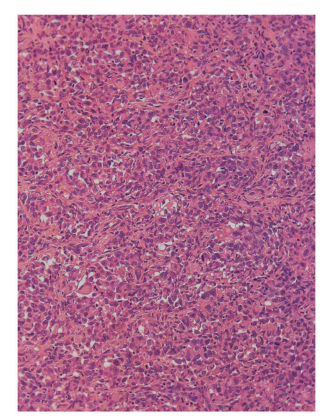

DTX commercial injection

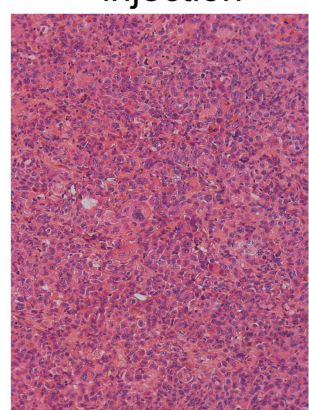

DTX-M

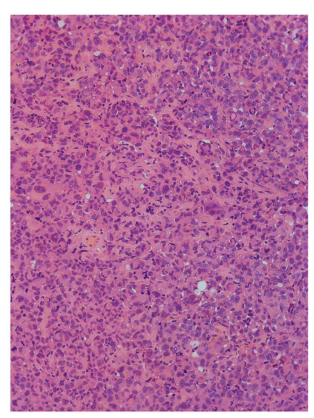

RGD-DTX-M

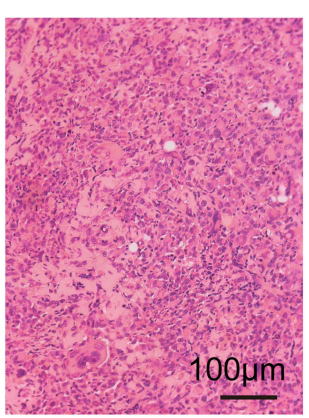

Figure 5 In vivo antitumor effects of RGD-DTX-M on MDA-MB-23I bearing nude mice. Tumor volume (A), body weight (B), tumor weight (C), tumor inhibitory rate (D), images of tumors $(\mathbf{E})$, and H\&E stain of tumors $(\mathbf{F})$ in each group after different treatments $\left(n=5, * p<0.05, *_{*} * *_{p}<0.00 \mathrm{I}\right)$. 
shown in Figure 5D, compared with DTX-M and DTX commercial injection, the tumor inhibition rate of RGDDTX-M was nearly 1.65-times and 5-times larger, respectively. In addition, the possible toxicity of RGDDTX-M was assayed by measuring body weight. According to the statistical analysis, during the whole experimental period there was no significant change in body weight of mice (Figure 5B). The damage of subcutaneous tumor tissue in different groups was also investigated by H\&E stain (Figure 5F). In the RGDDTX-M group, the most serious damage was observed, which suggested the best anti-tumor effect of RGD-DTX$\mathrm{M}$ among these experiment groups to MDA-MB-231 tumor in vivo. Overall, our results indicate that the RGD-modified DTX-M has a better antitumor effect than unmodified DTX-M and DTX commercial injections in vivo.

\section{Preliminary Safety Studies in vitro and in vivo}

In this study, human TNBC cell line MDA-MB-231 was used to assess the cytotoxicity of blank RGD-M in vitro. It can be seen from Figure 6A that at the blank RGD-M concentration of about $5 \mathrm{mg} / \mathrm{mL}$, the cell survival rate is above $85 \%$. And at the blank RGD-M concentration of 2 $\mathrm{mg} / \mathrm{mL}$, the cell survival rate is higher than $93 \%$. These in vitro study results demonstrated that a blank RGD-M concentration of $5 \mathrm{mg} / \mathrm{mL}$ had no obvious toxicity to MDA-MB-231 cells. Previous studies showed that the half lethal dose of the DTX commercial injection was $104.1 \mathrm{mg} / \mathrm{kg},{ }^{28}$ which was nearly 20 -times the dosage for the commercial preparation used in this experiment. Because of the low dose of DTX used in the antitumor effect study, the results showed there were no significant changes in terms of body weight among the five treatment groups during a span of 28 days (Figure 5C). Besides, the major organs H\&E-stained images of each group indicated that there was no noticeable symbol of organ damage (Figure 6B). These results suggest the good biocompatibility of the RGD-DTX-M delivery system.

\section{Discussion}

Currently, chemotherapy is one of the most important treatment strategies for TNBC.

As an effective chemotherapy drug for TNBC treatment, the therapeutic effect of DTX is impaired by its toxic effects, ${ }^{29}$ leading to poor efficacy of DTX in the treatment of TNBC. Besides, similar to many chemotherapeutic agents, DTX has a fairly low solubility in water and high lipophilicity, making it inconvenient for clinical application. Therefore, in order to enhance the efficacy of DTX for the treatment of TNBC, we use amphiphilic polymers (PEGDSPE) to prepare DTX lipid core micelles. Through modification by RGD, a new delivery system which has tumor blood vessel and tumor cell targeting ability was prepared so as to increase the solubility of DTX, improve targeting ability to tumor sites, reduce side-effects of DTX, and improve the therapeutic effect on TNBC.

As expected, the RGD-DTX-M with high encapsulation efficiency, strong targeting ability, and sustained release properties was easily prepared through film hydration method. In the in vitro cell-uptake experiment, the cell uptake of RGD-DTX-M increased gradually with the prolongation of incubation time, and was significantly higher than that of DTX-M at the same DTX concentration. Furthermore, the in vivo biodistribution experiment suggested that RGD-DiR-M could enrich at the tumor site through RGD targeting to the integrin receptor $\alpha_{v} \beta_{3}$ on the tumor surface. These results confirm that RGD-DTX$\mathrm{M}$ has a good tumor targeting ability, suggesting that surface modification successfully enhances cell internalization of micelles.

Drug delivery systems enter cells through different endocytosis pathways, including clathrin-mediated endocytosis, caveolin-mediated endocytosis, macropinocytosis, and phagocytosis. To clarify the cellular uptake mechanism of RGDDTX-M in MDA-MB-231 cells, the cells were treated with various endocytic inhibitors. Chlorpromazine can destroy the recycling of adaptor protein from the inner body, affect the assembly of the package depression, and inhibit the clathrin-mediated endocytosis. ${ }^{30}$ Filipin can bind to sterol and selectively inhibit caveolin-mediated endocytosis. ${ }^{31}$ Colchicine is a selective inhibitor of the macropinocytosis pathway. ${ }^{32}$ As a metabolic inhibitor, ${ }^{33}$ sodium azide can affect mitochondrial oxidative phosphorylation, consume ATP, and lead to energy supply disorder. BFA is a fungal metabolite, which can destroy the structure and function of Golgi apparatus. ${ }^{34}$ Monensin can promote $\mathrm{H}^{+}-\mathrm{K}^{+}$ion exchange and inhibit the formation of a lysosomal acid environment. $^{35}$ The results showed that the uptake of micelles by cells might be mediated by the caveolin, and the whole process involves the participation of Golgi body and acid lysosome, accompanied with the consumption of ATP. Li et $\mathrm{al}^{36}$ found the uptake mechanism of a RGDdecorated chitosan-functionalized single-walled carbon 
A

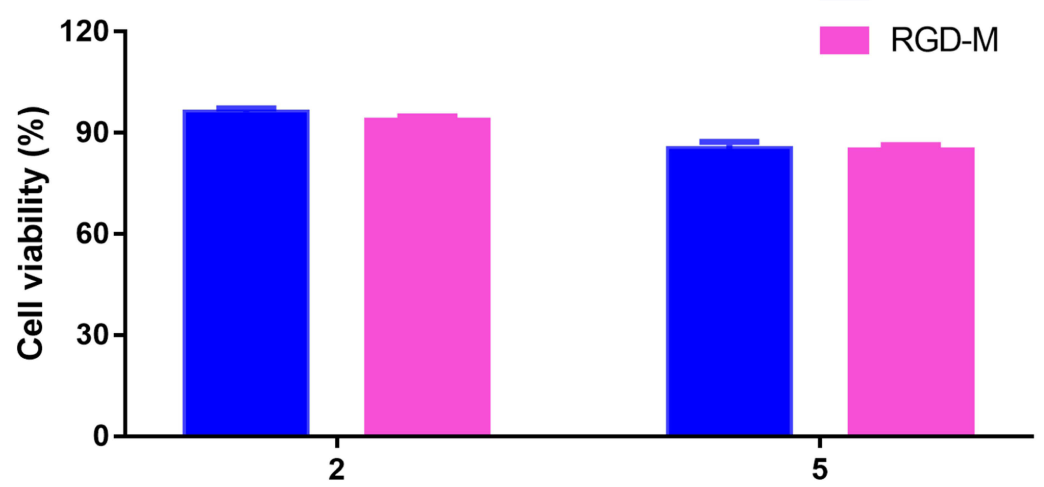

Micelle material concentration $(\mathrm{mg} / \mathrm{mL})$

B
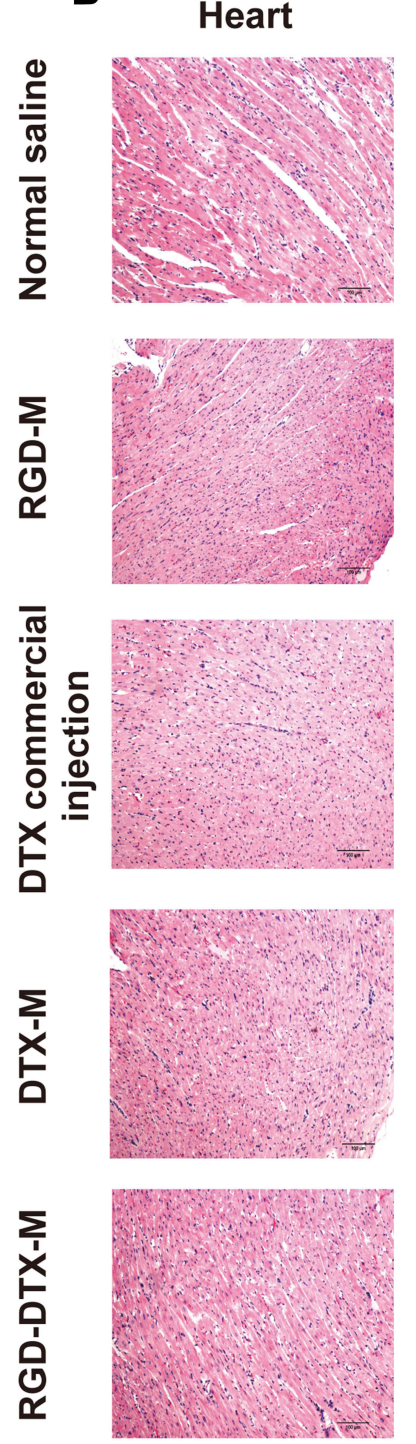

Liver
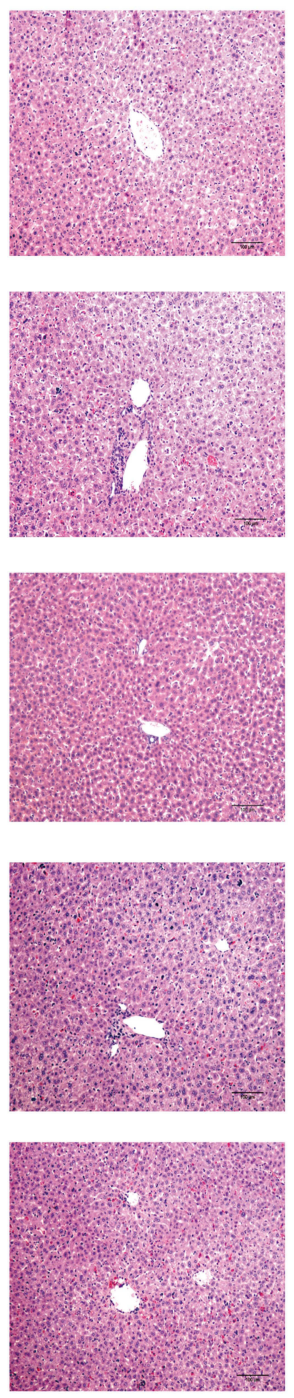

Spleen
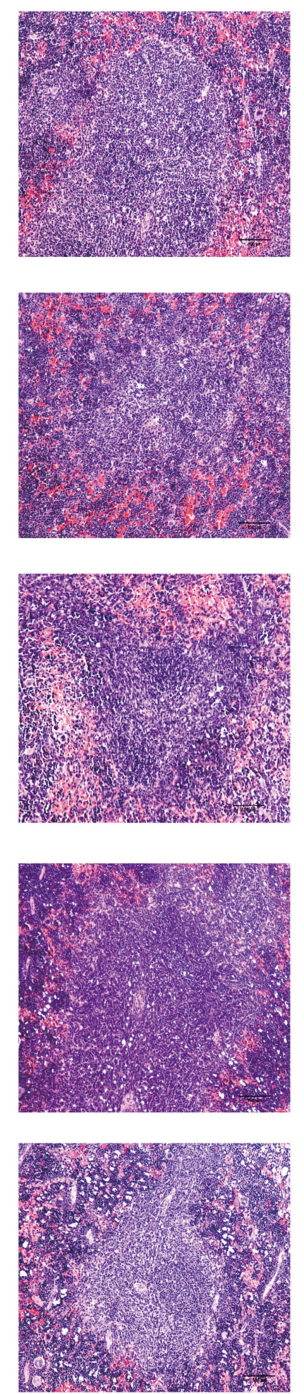

Lung
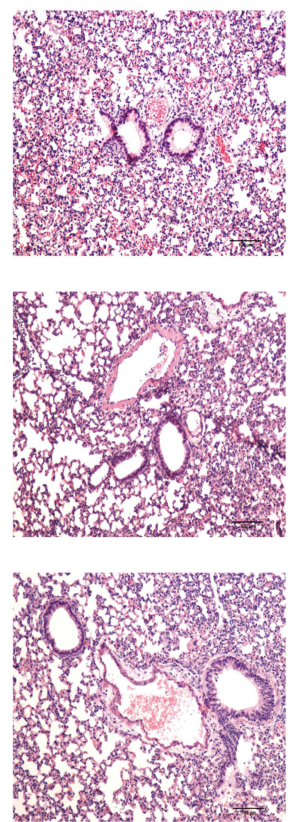

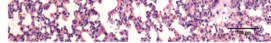
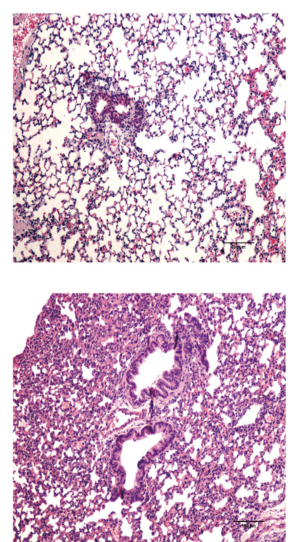

Kidney
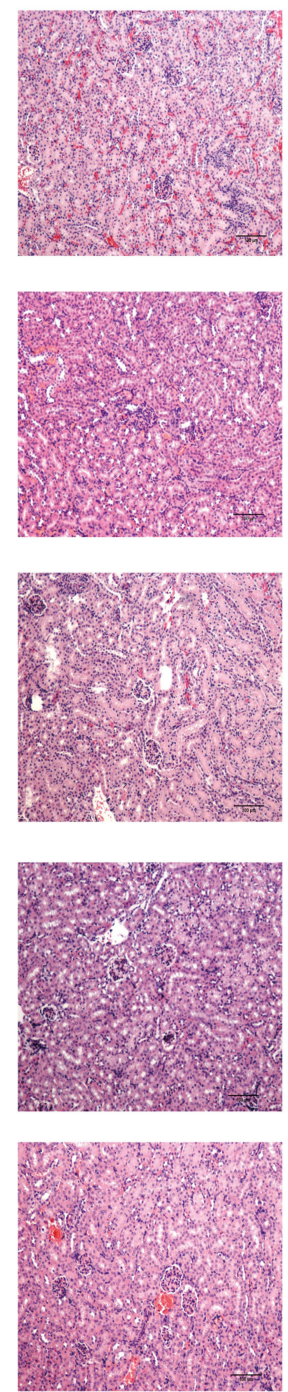

Figure 6 Preliminary safety studies of RGD-DTX-M in vitro and in vivo. (A) The effects of different concentrations of micelle materials on the survival rate of MDA-MB-23I cells $(n=4)$, (BMs, blank micelles). (B) H\&E staining of slices of major organs including hearts, livers, spleens, lungs, and kidneys from mice in different treatment groups ( $n=5$ ). 
nanotube was an energy-dependent, caveolin-mediated endocytosis process in MCF-7 cells. Liu et $\mathrm{al}^{37}$ also reported a RGD-modified dual-targeted theranostic photosensitizer (FL-RGD) could target tumor tissues and further locate in lysosomes of U87MG tumor cells. Our findings are consistent with these previous studies, suggesting that the uptake of RGD-modified micelles is a caveolin and energy-dependent endocytosis which involves the participation of the Golgi body and acid lysosome.

The pharmacokinetics of RGD-DTX-M was studied and compared with a DTX commercial injection in vivo. The AUC and TBC of RGD-DTX-M were 3.2- and 0.3times those of the commercial injection, respectively, which demonstrated that the RGD-M could improve the retention time of DTX in the blood and reduce the plasma clearance rate of DTX, which lead to the sustained release effect. Based on the sustained release effect of DTX and the powerful effect to target the tumor cells mediated by RGD, in pharmacodynamics experiments the RGD-DTX-M treatment reasonably led to the most significant anti-tumor effect of tumor xenografts. As shown in Figure 5, compared with the control group, all types of DTX formulations suppressed the human breast cancer (MDA-MB-231) growth and scaled down tumor size to a greater degree. However, the RGD-DTX-M treatment group had the most significant tumor volume reduction, compared with the other DTX formulation groups. Finally, in terms of the weight changes of mice during the whole experiment and the histological evaluation of animal organs by H\&E staining, RGD-DTX-M was preliminarily demonstrated to have favorable biocompatibility and safety. Thus, the novel RGD-DTX-M delivery system may be a promising formulation in enhancing the clinical efficacy of DTX for TNBC.

\section{Conclusion}

In summary (Figure 7), we constructed a novel RGDmodified lipid micelle delivery system to improve the therapeutic effects of DTX on TNBC. The preparation process of RGD-DTX-M was simple, which enabled large scale production of these formulations. The prepared RGD-DTX-M had high encapsulation efficiency, and showed sustained release property for intravenous administration. Cellular uptake experiments showed enhanced cellular accumulation of RGD-DTX-M which was mediated by the caveolin. The pharmacokinetics study results suggested a 3.2-times increased absolute bioavailability of RGD-DTX-M comparing with DTX commercial injections, which was accompanied with a 5-times increased antitumor-effect in MDA-MB-231 tumor-bearing mice. Preliminary safety results showed its good safety. The above results suggest that RGDDTX-M might be a novel anti-tumor formulation for triple negative breast cancer with high clinical application prospect.

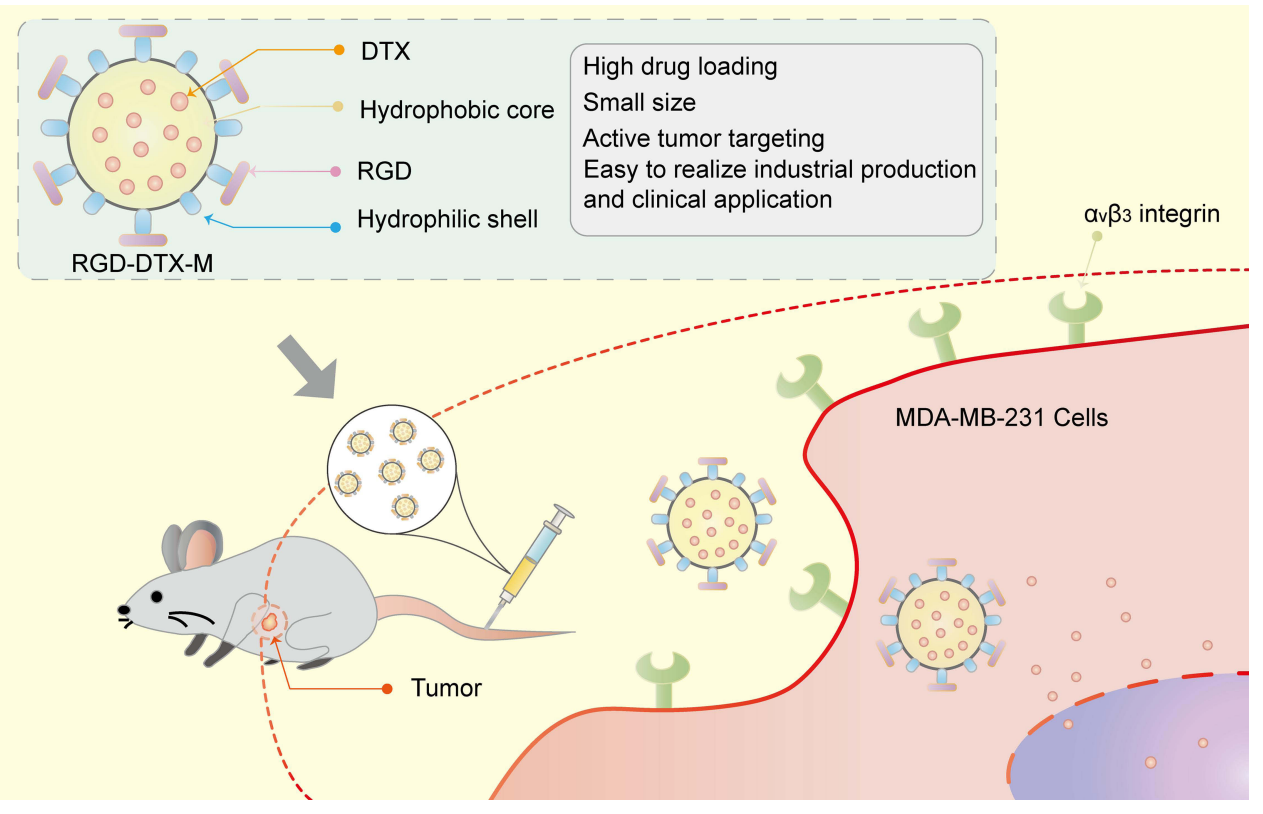

Figure 7 RGD-functionalized micelles (RGD-DTX-M) for highly potent and targeted TNBC chemotherapy. 


\section{Funding}

This work received financial supports from Nano-specific Project of Shanghai Science and Technology Commission (No. 1052nm05100, 11nm0506700), National Natural Science Foundation of China (No. 81202924, 81773909), Shanghai Rising-Star Program of China (No. 13QA1403400), Shanghai talent development funds (No. 201665), and Excellent Medical Profession Scholarship of Shanghai municipal commission of health and family planning (No. 2017YQ060).

\section{Disclosure}

The authors report no conflicts of interest in this work.

\section{References}

1. Chen L, Zhou L, Wang C, et al. Tumor-Targeted Drug and CpG Delivery System for Phototherapy and Docetaxel-Enhanced Immunotherapy with Polarization toward M1-Type Macrophages on Triple Negative Breast Cancers. Adv Mater. 2019;31(52):e1904997. doi:10.1002/adma.201904997

2. Varshosaz J, Enteshari S, Hassanzadeh F, Hashemi-Beni B, Minaiyan M, Mirsafaei R. Synthesis, in vitro characterization, and anti-tumor effects of novel polystyrene-poly(amide-ether-ester-imide) co-polymeric micelles for delivery of docetaxel in breast cancer in Balb/C mice. Drug Dev Ind Pharm. 2018;44(7):1139-1157. doi:10.1080/ 03639045.2018.1438462

3. Wen S, Manuel L, Doolan M, Westhuyzen J, Shakespeare TP, Aherne NJ. Effect of Clinical and Treatment Factors on Survival Outcomes of Triple Negative Breast Cancer Patients. Breast cancer. 2020;12:27-35. doi:10.2147/BCTT.S236483

4. Sumera Anwar A, Ovais M, Khan A, Raza A. Docetaxel-loaded solid lipid nanoparticles: a novel drug delivery system. IET Nanobiotechnol. 2017;11(6):621-629. doi:10.1049/iet-nbt.2017.0001

5. Ashrafizadeh M, Ahmadi Z, Mohamadi N, et al. Chitosan-based advanced materials for docetaxel and paclitaxel delivery: recent advances and future directions in cancer theranostics. Int $J$ Biol Macromol. 2020;145:282-300. doi:10.1016/j.ijbiomac.2019.12.145

6. Li Y, Zhang H, Zhai GX. Intelligent polymeric micelles: development and application as drug delivery for docetaxel. J Drug Target. 2017;25(4):285-295. doi:10.1080/1061186X.2016.1245309

7. Li J, Yu K, Pang D, et al. Adjuvant Capecitabine With Docetaxel and Cyclophosphamide Plus Epirubicin for Triple-Negative Breast Cancer (CBCSG010): an Open-Label, Randomized, Multicenter, Phase III Trial. J Clin Oncol Off J Am Soc Clin Oncol. 2020;38 (16):1774-1784. doi:10.1200/JCO.19.02474

8. Rodallec A, Brunel JM, Giacometti S, et al. Docetaxel-trastuzumab stealth immunoliposome: development and in vitro proof of concept studies in breast cancer. Int $J$ Nanomedicine. 2018;13:3451-3465. doi: $10.2147 /$ IJN.S162454

9. Engels FK, Mathot RAA, Verweij J. Alternative drug formulations of docetaxel: a review. Anticancer Drugs. 2007;18(2):95-103. doi:10.1097/CAD.0b013e3280113338

10. Cheng YA, Chen IJ, Su YC, et al. Enhanced drug internalization and therapeutic efficacy of PEGylated nanoparticles by one-step formulation with anti-mPEG bispecific antibody in intrinsic drug-resistant breast cancer. Biomater Sci. 2019;7(8):3404-3417. doi:10.1039/c9bm00323a

11. Zhang Y, Guo Z, Cao Z, et al. Endogenous albumin-mediated delivery of redox-responsive paclitaxel-loaded micelles for targeted cancer therapy. Biomaterials. 2018;183:243-257. doi:10.1016/j.biomaterials. 2018.06.002
12. Gustafson HH, Holt-Casper D, Grainger DW, Ghandehari H. Nanoparticle uptake: the phagocyte problem. Nano Today. 2015;10 (4):487-510. doi:10.1016/j.nantod.2015.06.006

13. Shi J, Kantoff PW, Wooster R, Farokhzad OC. Cancer nanomedicine: progress, challenges and opportunities. Nat Rev Cancer. 2017;17 (1):20-37. doi:10.1038/nrc.2016.108

14. Sun L, Wang D, Chen Y, et al. Core-shell hierarchical mesostructured silica nanoparticles for gene/chemo-synergetic stepwise therapy of multidrug-resistant cancer. Biomaterials. 2017;133:219-228. doi:10. 1016/j.biomaterials.2017.04.028

15. Yang G, Liu Y, Wang H, et al. Bioinspired Core-Shell Nanoparticles for Hydrophobic Drug Delivery. Angew Chem Int Ed Engl. 2019;58 (40):14357-14364. doi:10.1002/anie.201908357

16. Fattahi N, Shahbazi MA, Maleki A, Hamidi M, Ramazani A, Santos HA. Emerging insights on drug delivery by fatty acid mediated synthesis of lipophilic prodrugs as novel nanomedicines. $J$ Control Release. 2020;326:556-598. doi:10.1016/j.jconrel.2020. 07.012

17. Zhao Y, Duan S, Zeng X, et al. Prodrug strategy for PSMA-targeted delivery of TGX-221 to prostate cancer cells. Mol Pharm. 2012;9 (6):1705-1716. doi:10.1021/mp3000309

18. Qi J, Hu X, Dong X, et al. Towards more accurate bioimaging of drug nanocarriers: turning aggregation-caused quenching into a useful tool. Adv Drug Deliv Rev. 2019;143:206-225. doi:10.1016/j. addr.2019.05.009

19. Fedele M, Cerchia L, Chiappetta G. The epithelial-to-mesenchymal transition in breast cancer: focus on basal-like carcinomas. Cancers. 2017;9(10):134. doi:10.3390/cancers9100134

20. Song Y, Guo X, Fu J, et al. Dual-targeting nanovesicles enhance specificity to dynamic tumor cells in vitro and in vivo via manipulation of avp3-ligand binding. Acta Pharm Sin B. 2020;10(11):21832197. doi:10.1016/j.apsb.2020.07.012

21. Rathinam R, Alahari SK. Important role of integrins in the cancer biology. Cancer Metastasis Rev. 2010;29(1):223-237. doi:10.1007/ s10555-010-9211-x

22. Othman BA, Greenwood C, Abuelela AF, et al. Correlative LightElectron Microscopy Shows RGD-Targeted ZnO Nanoparticles Dissolve in the Intracellular Environment of Triple Negative Breast Cancer Cells and Cause Apoptosis with Intratumor Heterogeneity. Adv Healthc Mater. 2016;5(11):1310-1325. doi:10.1002/adhm.20 1501012

23. Gao X, Yang H, Wu M, et al. Targeting Delivery of Lidocaine and Cisplatin by Nanogel Enhances Chemotherapy and Alleviates Metastasis. ACS Appl Mater Interfaces. 2018;10(30):25228-25240. doi:10.1021/acsami.8b09376

24. Jin G, He R, Liu Q, et al. Theranostics of Triple-Negative Breast Cancer Based on Conjugated Polymer Nanoparticles. ACS Appl Mater Interfaces. 2018;10(13):10634-10646. doi:10.1021/acsami. $7 \mathrm{~b} 14603$

25. Zhu R, Tian Y. Preparation and evaluation of RGD and TAT comodified docetaxel-loaded liposome. Drug Des Devel Ther. 2017;11:3481-3489. doi:10.2147/DDDT.S149620

26. Xiao W, Xiong J, Zhang S, Xiong Y, Zhang H, Gao H. Influence of ligands property and particle size of gold nanoparticles on the protein adsorption and corresponding targeting ability. Int J Pharm. 2018;538 (1-2):105-111. doi:10.1016/j.ijpharm.2018.01.011

27. Pu Y, Zhang H, Peng Y, et al. Dual-targeting liposomes with active recognition of GLUT(5) and $\alpha(\mathrm{v}) \beta(3)$ for triple-negative breast cancer. Eur J Med Chem. 2019;183:111720. doi:10.1016/j.ejmech.20 19.111720

28. Xu HL, Mao KL, Lu CT, et al. An injectable acellular matrix scaffold with absorbable permeable nanoparticles improves the therapeutic effects of docetaxel on glioblastoma. Biomaterials. 2016;107:44-60. doi:10.1016/j.biomaterials.2016.08.026

29. Steeg PS. Tumor metastasis: mechanistic insights and clinical challenges. Nat Med. 2006;12(8):895-904. doi:10.1038/nm1469 
30. Wang J, Gou W, Dosung K, Strange C, Wang H. Clathrin-mediated Endocytosis of Alpha-1 Antitrypsin is Essential for its Protective Function in Islet Cell Survival. Theranostics. 2019;9(13):39403951. doi: $10.7150 /$ thno. 31647

31. Yamano S, Dai J, Hanatani S, et al. Long-term efficient gene delivery using polyethylenimine with modified Tat peptide. Biomaterials. 2014;35(5):1705-1715. doi:10.1016/j.biomaterials.2013.11.012

32. Linares J, Matesanz MC, Vila M, et al. Endocytic mechanisms of graphene oxide nanosheets in osteoblasts, hepatocytes and macrophages. ACS Appl Mater Interfaces. 2014;6(16):13697-13706. doi:10.1021/am5031598

33. Zhang C, Xue J, Cheng D, et al. Uptake, translocation and distribution of three veterinary antibiotics in Zea mays L. Environ Pollut. 2019;250:47-57. doi:10.1016/j.envpol.2019.03.110
34. Van Damme T, Gardeitchik T, Mohamed M, et al. Mutations in ATP6V1E1 or ATP6V1A Cause Autosomal-Recessive Cutis Laxa. Am J Hum Genet. 2017;100(2):216-227. doi:10.1016/j.ajhg.2016.12.010

35. Janikiewicz J, Hanzelka K, Dziewulska A, et al. Inhibition of SCD1 impairs palmitate-derived autophagy at the step of autophagosomelysosome fusion in pancreatic $\beta$-cells. J Lipid Res. 2015;56 (10):1901-1911. doi:10.1194/jlr.M059980

36. Li B, Zhang XX, Huang HY, et al. Effective deactivation of A549 tumor cells in vitro and in vivo by RGD-decorated chitosan-functionalized single-walled carbon nanotube loading docetaxel. Int $J$ Pharm. 2018;543(1-2):8-20. doi:10.1016/j.ijpharm.2018.03.017

37. Liu Z, Shi W, Hong G, et al. A dual-targeted theranostic photosensitizer based on a TADF fluorescein derivative. J Control Release. 2019;310:1-10. doi:10.1016/j.jconrel.2019.08.001

\section{Publish your work in this journal}

The International Journal of Nanomedicine is an international, peerreviewed journal focusing on the application of nanotechnology in diagnostics, therapeutics, and drug delivery systems throughout the biomedical field. This journal is indexed on PubMed Central, MedLine, CAS, SciSearch ${ }^{\mathbb{R}}$, Current Contents ${ }^{\mathbb{R}} /$ Clinical Medicine,
Journal Citation Reports/Science Edition, EMBase, Scopus and the Elsevier Bibliographic databases. The manuscript management system is completely online and includes a very quick and fair peer-review system, which is all easy to use. Visit http://www.dovepress.com/ testimonials.php to read real quotes from published authors. 